\title{
A LOCAL CHARACTERIZATION OF VC-MINIMALITY
}

\author{
URI ANDREWS AND VINCENT GUINGONA
}

(Communicated by Mirna Džamonja)

\begin{abstract}
We show VC-minimality is $\Pi_{4}^{0}$-complete. In particular, we give a local characterization of VC-minimality. We also show dp-smallness is $\Pi_{1}^{1}$ complete.
\end{abstract}

\section{INTRODUCTION}

Motivated by successes in stability theory, model theorists have recently been interested in generalizing results for stable theories to a wider class of theories. The primary candidate of study is the class of NIP theories 11 which includes mathematically important theories left out of the class of stable theories, such as the first-order theory of the real field and the $p$-adic field. To study this large class of theories, it helps to first understand simpler cases, considering theories that are minimal with respect to various notions of "dimension" (e.g., Vapnik-Chervonenkis (VC) dimension and dp-rank).

VC-minimality is a notion of simplicity for a first-order theory which simultaneously generalizes weak o-minimality and C-minimality. Until now, VC-minimality has been a very difficult notion to work with. This difficulty is due to the complexity of the definition of VC-minimality. In particular, the definition is $\Sigma_{1}^{1}$, i.e., it requires an existential quantifier over sets of formulae. As such, it is quite difficult to verify that a theory is not VC-minimal. Instead, most instances of proofs that a theory is not VC-minimal actually show that the theory fails to satisfy one of several weaker principles such as convex orderability, dp-smallness, or dp-minimality. In this paper, we answer the following question:

Question 1.1. How hard is it to determine whether or not a theory is VC-minimal?

Index sets are a tool used to quantify the complexity of notions. Let $P$ be a property of objects in a class $K$. Then the index set of $P$ is the set

$$
I(P):=\{i \mid i \text { is an index for a recursive } C \in K \text { with the property } P\} .
$$

By restricting to the recursive $C \in K$, the complexity of this set comes from the complexity of the notion $P$, not the inherent complexity in the object $C$.

The question is formalized as asking to characterize the complexity of the index set $I$ (VC-minimal theories). We show that in fact VC-minimality is far simpler

Received by the editors July 22, 2014 and, in revised form, March 21, 2015.

2010 Mathematics Subject Classification. Primary 03C45, 03C57, 03D80.

The first author's research was partially supported by NSF grant DMS-1201338. The second author's research was supported by NSF grant DMS-0838506. This material is based upon work supported by the NSF under grant no. 0932078000 while both authors were in residence at the Mathematical Sciences Research Institute in Berkeley, California, during the Spring 2014 semester.

1 "NIP" stands for "Not the Independence Property". 
than expected, being $\Pi_{4}^{0}$-complete, and we give a simple characterization. This characterization makes VC-minimality far easier work with. We hope that it will spur further research in the area. Our characterization, which holds for theories in countable languages, is "local" in the sense that it gives a condition that must hold for each formula.

It is known that VC-minimality implies convex orderability [7, which in turn implies dp-smallness [6] (which implies dp-minimality which implies NIP). So, a question naturally arises: How complex are the definitions of convex orderability and dp-smallness?

We show that dp-smallness is, in fact, far more complicated than VC-minimality; it is $\Pi_{1}^{1}$-complete. In this vein, we also answer a question from [6] by giving examples of dp-small theories in countable languages which are not convexly orderable.

We leave the following question open:

Open question 1.2. What is the complexity of convex orderability?

\section{BACKGROUND}

Let $X$ be a set, $\mathcal{C} \subseteq \mathcal{P}(X)$. We say $\mathcal{C}$ is directed if, for all $A, B \in \mathcal{C}$, at least one of the following holds:

- $A \subseteq B$,

- $B \subseteq A$, or

- $A \cap B=\emptyset$.

For simplicity of notation, for $A, B \subseteq X$, we write $A \perp B$ to denote that $\{A, B\}$ is not directed. That is,

- $A \backslash B \neq \emptyset$,

- $B \backslash A \neq \emptyset$, and

- $A \cap B \neq \emptyset$.

Remark 2.1 (Swiss cheese decomposition). Suppose $\mathcal{C} \subseteq \mathcal{P}(X)$ is directed. If $A \in \mathcal{C}$ and $B_{i} \in \mathcal{C}$ for $i<n$ with $B_{i} \subseteq A$ for all $i<n$ and $B_{i} \cap B_{j}=\emptyset$ for all $i \neq j$, then we call $S=A \backslash\left(B_{0} \cup \cdots \cup B_{n-1}\right)$ a Swiss cheese; $A$ is the wheel of $S$ and the $B_{i}$ 's are the holes of $S$. If $D \subseteq X$ is a (finite) boolean combination of elements of $\mathcal{C}$, then there exists Swiss cheeses $S_{0}, \ldots, S_{m-1}$ such that

- $S_{i} \cap S_{j}=\emptyset$ for all $i \neq j$,

- no wheel of some $S_{i}$ is equal to a hole of some $S_{j}$, and

- $D=S_{0} \cup \cdots \cup S_{m-1}$.

We call such $S_{0}, \ldots, S_{m-1}$ a Swiss cheese decomposition of $D$. See Lemma 2.1 of [4] for more details. By Theorem 3.1 of [4], there is a means of canonically choosing a decomposition, so we may consider "the" Swiss cheese decomposition of $D$.

Lemma 2.2 (Union of chains). If $\mathcal{C} \subseteq \mathcal{P}(X)$ is directed, $\mathcal{C}_{0} \subseteq \mathcal{C}$ is a chain, and $A:=\bigcup \mathcal{C}_{0}$, then $\mathcal{C} \cup\{A\}$ is directed.

Proof. Fix $B \in \mathcal{C}$. We must show that either $A \cap B=\emptyset, A \subseteq B$, or $B \subseteq A$. If any $B^{\prime} \in \mathcal{C}_{0}$ contains $B$, then $B \subseteq A$, and we are done. Similarly, if every $B^{\prime} \in \mathcal{C}_{0}$ is disjoint from $B$, then $A \cap B=\emptyset$. The remaining case is where some $B^{\prime} \in \mathcal{C}_{0}$ intersects $B$, but none contains $B$. Thus this $B^{\prime}$ is contained in $B$. As $\mathcal{C}_{0}$ is a chain, every element intersects $B$ and none contains $B$, so every member of the chain is contained in $B$. Thus $A \subseteq B$. 
Definition 2.3. Fix a language $L$, an $L$-theory $T$, and a monster model $\mathcal{U} \models T$. For a tuple of variables $x$, let $|x|$ denote the length of $x$ and let $\mathcal{U}_{x}=\mathcal{U}^{|x|}$. If $\varphi(x ; y)$ is a formula and $b \in \mathcal{U}_{y}$, then let $\varphi(\mathcal{U} ; b)$ be the set of all $a \in \mathcal{U}_{x}$ satisfying the formula $\varphi(a ; b)$.

- We say a set of partitioned $L$-formulae $\Psi=\left\{\psi_{i}\left(x ; y_{i}\right) \mid i \in I\right\}$ is directed if the set $\mathcal{C}_{\Psi}:=\left\{\psi_{i}(\mathcal{U} ; b) \mid i \in I, b \in \mathcal{U}_{y_{i}}\right\}$ is directed (in the ambient set $\mathcal{U}_{x}$ ).

- We say that the theory $T$ is $V C$-minimal if there exists a directed set of formulae $\Psi$ (in the free variable $x$ with $|x|=1$ ) such that every (parameter) definable set $A \subseteq \mathcal{U}$ is a (finite) boolean combination of elements from $\mathcal{C}_{\Psi}$.

- In this case, we call $\Psi$ a generating family for $T$.

- If $\Psi$ is a generating family for $T$, then a set $\psi_{i}(\mathcal{U}, b)$ for $\psi_{i} \in \Psi$ is called a ball in $\Psi$.

If $\mathcal{C} \subseteq \mathcal{P}(X)$ is directed, then $\mathcal{C} \cup\{\{a\} \mid a \in X\}$ is directed. Therefore, without loss of generality, we may assume the formula $x=y$ is in the generating family of any $\mathrm{VC}$-minimal theory.

An $L$-structure $M$ is called convexly orderable if there exists a linear order $\unlhd$ on $M$ (not necessarily definable) such that, for all $L$-formulas $\varphi(x ; y)$ with $|x|=1$, there exists $k<\omega$ such that, for all $b \in M_{y}, \varphi(M ; b)$ is a union of at most $k$ $\unlhd$-convex subsets of $M$. By Proposition 2.3 of [7], if $M$ is convexly orderable and $N \equiv M$, then $N$ is convexly orderable, so convex orderability is a property of theories. By Theorem 2.4 of [7], any VC-minimal theory is convexly orderable.

\section{Devastation And immortality}

The following is a technical definition which plays an important role in our local characterization of VC-minimality.

Definition 3.1 (Devastation, immortality). Suppose that $\psi(x ; y)$ is a partitioned $L$-formula and $\varphi(x)$ is an $L(\mathcal{U})$-formula, both with a common free variable, $x$. We say that $\varphi$ devastates $\psi$ if there exists a sequence $\left\langle c_{i}: i<\omega\right\rangle$ of elements in $\mathcal{U}_{y}$ such that, for all $i<j<\omega$,

$$
\begin{aligned}
& \text { - } \models \exists x\left(\psi\left(x ; c_{i}\right) \wedge \neg \psi\left(x ; c_{j}\right) \wedge \varphi(x)\right) \text {, and } \\
& \text { - } \models \exists x\left(\psi\left(x ; c_{i}\right) \wedge \neg \psi\left(x ; c_{j}\right) \wedge \neg \varphi(x)\right) .
\end{aligned}
$$

If there exists no $L(\mathcal{U})$-formula $\varphi(x)$ which devastates $\psi(x ; y)$, then we say that $\psi(x ; y)$ is immortal.

Remark 3.2. If $\varphi(x ; y)$ is an $L$-formula such that, for all $d \in \mathcal{U}_{y}, \varphi(x ; d)$ does not devastate $\psi(x ; z)$, then by compactness there exists $k<\omega$ such that, for all $d \in \mathcal{U}_{y}$, there does not exist $\left\langle c_{i}: i<k\right\rangle$ from $\mathcal{U}_{z}$, so that for all $i<j<k$,

- $\models \exists x\left(\psi\left(x ; c_{i}\right) \wedge \neg \psi\left(x ; c_{j}\right) \wedge \varphi(x, d)\right)$, and

- $\models \exists x\left(\psi\left(x ; c_{i}\right) \wedge \neg \psi\left(x ; c_{j}\right) \wedge \neg \varphi(x, d)\right)$.

It follows that immortality of $\psi(x, y)$ in a recursive theory $T$ is a $\Pi_{2}^{0}$ condition defined by $\forall \varphi(x ; y) \exists k T \vdash \theta$ where $\theta$ is the sentence saying there are no $\left\langle c_{i}: i<k\right\rangle$ as above.

If $\varphi(x)$ devastates $\psi(x ; y)$ witnessed by $\bar{c}:=\left\langle c_{i}: i<\omega\right\rangle$, then we may assume $\bar{c}$ is indiscernible (i.e., any two finite subsequences of $\bar{c}$ satisfy all the same $L$-formulae). To see this, use compactness to reduce to only finitely many $L$-formulae and a finite length sequence, then use Ramsey's Theorem to choose a finite subsequence of $\bar{c}$ where all the selected $L$-formulae either hold or fail similarly across the subsequence. 
Lemma 3.3 (Directed and devastated). Suppose $\varphi(x)$ is an $L(\mathcal{U})$-formula, and $\psi(x ; y)$ is a directed $L$-formula. Then $\varphi(x)$ devastates $\psi(x ; y)$ if and only if there exists an indiscernible sequence $\left\langle c_{i}: i<\omega\right\rangle$ in $\mathcal{U}_{y}$ such that one of the following holds:

(1) for all $i<\omega, \models \forall x\left(\psi\left(x ; c_{i+1}\right) \rightarrow \psi\left(x ; c_{i}\right)\right), \models \exists x\left(\psi\left(x ; c_{i}\right) \wedge \neg \psi\left(x ; c_{i+1}\right) \wedge\right.$ $\varphi(x))$, and $\models \exists x\left(\psi\left(x ; c_{i}\right) \wedge \neg \psi\left(x ; c_{i+1}\right) \wedge \neg \varphi(x)\right)$; or

(2) for all $i<\omega, \models \neg \exists x\left(\psi\left(x ; c_{i+1}\right) \wedge \psi\left(x ; c_{i}\right)\right), \models \exists x\left(\psi\left(x ; c_{i}\right) \wedge \varphi(x)\right)$, and $\vDash \exists x\left(\psi\left(x ; c_{i}\right) \wedge \neg \varphi(x)\right)$.

Proof. If (1) or (2) hold, then clearly $\varphi(x)$ devastates $\psi(x ; y)$. Conversely, if $\varphi(x)$ devastates $\psi(x ; y)$, then by Remark 3.2 we can assume the witness $\left\langle c_{i}: i\langle\omega\rangle\right.$ is indiscernible. Therefore, we have either that, for all $i<\omega, \psi\left(\mathcal{U} ; c_{i+1}\right) \subseteq \psi\left(\mathcal{U} ; c_{i}\right)$, or for all $i<j<\omega, \psi\left(\mathcal{U} ; c_{i}\right) \cap \psi\left(\mathcal{U} ; c_{j}\right)=\emptyset$. Now (1) or (2) follow from each case.

Definition 3.4. If $\varphi(x ; y)$ is any formula and $a$ is any parameter, we refer to $\varphi(x ; a)$ as an instance of $\varphi$.

Definition 3.5 (Instance sums). Fix $L$-formulae $\varphi(x ; y)$ and $\psi(x ; z)$. Then their instance sum is the following formula:

$$
(\varphi \oplus \psi)\left(x ; y, z, w_{0}, w_{1}\right):=\left(w_{0}=w_{1} \rightarrow \varphi(x ; y)\right) \wedge\left(w_{0} \neq w_{1} \rightarrow \psi(x ; z)\right) .
$$

Remark 3.6 (On instance sums). If $\varphi(x ; y)$ and $\psi(x ; z)$ are $L$-formulae, then each instance of $(\varphi \oplus \psi)$ is $T$-equivalent to either an instance of $\varphi$ or an instance of $\psi$. Conversely, each instance of $\varphi$ and each instance of $\psi$ is $T$-equivalent to an instance of $(\varphi \oplus \psi)$.

If $\varphi(x ; y)$ and $\psi(x ; z)$ are immortal $L$-formulae, then $(\varphi \oplus \psi)$ is immortal. If $\delta(x)$ devastates $(\varphi \oplus \psi)$, then by the pigeonhole principle, either $\delta$ devastates $\varphi$ or $\delta$ devastates $\psi$. This contradicts the assumption that both formulae are immortal.

If $\{\varphi(x ; y), \psi(x ; z)\}$ is directed, then $(\varphi \oplus \psi)$ is directed.

Lemma 3.7 (Balls are immortal). If $T$ is $V C$-minimal and $\psi(x ; z)$ is in the generating family of $T$, then $\psi$ is immortal.

Proof. Suppose, by means of contradiction, that $\psi(x ; z)$ is in the generating family of $T$ but $\psi$ is not immortal. Therefore, there exists an $L(\mathcal{U})$-formula $\varphi(x)$ which devastates $\psi$. Then by Lemma 3.3. there are $a_{i}, b_{i} \in \mathcal{U}_{x}$ and $c_{i} \in \mathcal{U}_{z}$ such that, for all $i<j<\omega$,

- $a_{i} \in \varphi(\mathcal{U}) \cap \psi\left(\mathcal{U} ; c_{i}\right) \backslash \psi\left(\mathcal{U} ; c_{j}\right)$, and

- $b_{i} \in \neg \varphi(\mathcal{U}) \cap \psi\left(\mathcal{U} ; c_{i}\right) \backslash \psi\left(\mathcal{U} ; c_{j}\right)$.

Since $T$ is VC-minimal, $\varphi(\mathcal{U})$ has a Swiss cheese decomposition, namely $S_{0}, \ldots, S_{m-1}$ as in Remark 2.1. Therefore, by the pigeonhole principle, for some $j<m$ we have infinitely many $i<\omega$ such that $a_{i} \in S_{j}$. Let $S=S_{j}$ and, without loss of generality, suppose all $a_{i} \in S$. Let $A$ be the wheel and $B_{0}, \ldots, B_{m-1}$ be the holes of $S$ (if $S$ has no holes, we get a contradiction, since $\psi\left(\mathcal{U} ; c_{i}\right) \nsubseteq S$ for any $\left.i<\omega\right)$. By the pigeonhole principle again, there exists $j<m$ and infinitely many $i<\omega$ such that $b_{i} \in B_{j}$. Let $B=B_{j}$. For each $i \geq 1$ : since $b_{i} \in B, B \cap \psi\left(\mathcal{U}, c_{i}\right) \neq \emptyset$. Since $b_{i-1} \in B, B \nsubseteq \psi\left(\mathcal{U}, c_{i}\right)$. Thus $\psi\left(\mathcal{U}, c_{i}\right) \subseteq B$. But now $a_{i} \in B$, so $a_{i} \notin S$, which contradicts our choice of $S$. 


\section{Local Characterization of VC-Minimality}

Theorem 4.1 (Local characterization of VC-minimality). For a theory $T$ in a countable language $L$, the following are equivalent:

(1) $T$ is VC-minimal,

(2) for all L-formulae $\varphi(x ; y)$, there exists an immortal directed L-formula $\psi(x ; z)$ such that each instance of $\varphi$ is T-equivalent to a (finite) boolean combination of instances of $\psi$.

Since compactness shows that if every instance of $\varphi$ is equivalent to a boolean combination of instances of $\psi$, then there is an $n$ so that every instance of $\varphi$ is a boolean combination of $\leq n$ instances of $\psi$; this shows that the index set of VC-minimal theories is $\Pi_{4}^{0}$.

Remark 4.2. Our restriction to a countable language is necessary. Consider the example in the language $L=\left\{P_{i} \mid i<\omega_{1}\right\}$ with $\aleph_{1}$-many unary predicates and let $T$ be the $L$-theory which says that, for all finite disjoint $I, J \subseteq \omega_{1}$, there are infinitely many $x$ such that

$$
\bigwedge_{i \in I} P_{i}(x) \wedge \bigwedge_{j \in J} \neg P_{j}(x)
$$

This theory has quantifier elimination and is superstable. One can easily check that it satisfies condition (2) of Theorem 4.1] but this is not VC-minimal (see Example 2.10 of [7] for more details).

Lemma 4.3 (Main Construction Lemma). If $\varphi(x ; y)$ and $\psi(x ; z)$ are each a directed immortal formula (not assuming $\{\varphi, \psi\}$ is directed), then there exists $\delta(x ; w)$ an immortal formula such that

- $\{\psi, \delta\}$ is directed, and

- each instance of $\varphi$ is a finite boolean combination of instances of $\psi$ and $\delta$.

As the proof of the Main Construction Lemma is somewhat involved and combinatorial, we leave it to Section 8 . We now consider the proof of Theorem 4.1, given the Main Construction Lemma.

Proof of Theorem 4.1. (1) $\Rightarrow(2)$ : Suppose $T$ is VC-minimal and fix an $L$-formula $\varphi(x ; y)$. By compactness, there exists a directed family of finitely many $L$-formulae $\left\{\psi_{i}\left(x ; z_{i}\right) \mid i<k\right\}$ such that each instance of $\varphi$ is $T$-equivalent to a boolean combination of instances of the $\psi_{i}$ 's. By taking instance sums, we may assume that $k=1$. By Lemma 3.7, $\psi$ is immortal.

$(2) \Rightarrow(1)$ : We construct $\Psi$ the generating family by induction. First, since $L$ is countable, there exists an enumeration $\left\{\varphi_{i}\left(x ; y_{i}\right) \mid i<\omega\right\}$ of the $L$-formulae with $x$ (where $|x|=1$ ) as a free variable. Let $\Psi_{0}=\emptyset$ and suppose that we have $\Psi_{i}$ a finite directed set of immortal $L$-formulae constructed so that, for all $j<i$, each instance of $\varphi_{j}$ is $T$-equivalent to a boolean combination of instances of elements from $\Psi_{j+1}$. Suppose further that $\Psi_{j} \subseteq \Psi_{j+1}$ for all $j<i$. Now consider $\varphi_{i}\left(x ; y_{i}\right)$ and let $\psi(x ; z)$ be given as in (2) (hence $\psi$ is immortal and directed). Let $\psi^{\prime}\left(x ; z^{\prime}\right)$ be the instance sum of $\Psi_{i}$, which is immortal and directed by Remark [3.6. By Lemma 4.3, there exists $\delta(x ; w)$ an immortal $L$-formula such that $\left\{\delta, \psi^{\prime}\right\}$ is directed and each instance of $\psi$ is $T$-equivalent to a boolean combination of instances of $\psi^{\prime}$ and $\delta$. Therefore, each instance of $\varphi$ is $T$-equivalent to a boolean combination 
of instances of $\psi^{\prime}$ and $\delta$. Let $\Psi_{i+1}:=\Psi_{i} \cup\{\delta\}$, which is a finite directed set of immortal $L$-formulae. Finally, let $\Psi=\bigcup_{i} \Psi_{i}$.

\section{Stable VC-Minimal theories}

A formula $\varphi(x ; y)$ has the order property if there exists elements $a_{i} \in \mathcal{U}_{x}$ for $i<\omega$ and $b_{j} \in \mathcal{U}_{y}$ for $j<\omega$ such that $\varphi\left(a_{i} ; b_{j}\right)$ if and only if $i<j$. A theory $T$ is stable if no formula has the order property.

Lemma 5.1. Suppose $T$ is VC-minimal and stable. Then there exists

$$
\Psi:=\left\{E_{i}(x, y) \mid i \in I\right\}
$$

a directed set of equivalence relations (on $x$ with $|x|=1$ ) that is a generating family for $T$.

Proof. Since $T$ is VC-minimal, let $\Psi^{\prime}$ be a generating family for $T$. Now fix $\psi(x ; y) \in$ $\Psi^{\prime}$ and $p(y) \in S_{y}(\emptyset)$ (i.e., $p$ is a maximally consistent set of $L$-formulae with free variable $y$ ). Suppose, by means of contradiction, that the type

$$
p\left(y_{0}\right) \cup p\left(y_{1}\right) \cup\left\{\psi\left(\mathcal{U} ; y_{0}\right) \subsetneq \psi\left(\mathcal{U} ; y_{1}\right)\right\}
$$

is consistent. Take $\left\langle b_{0}, b_{1}\right\rangle$ a witness to this and take $\sigma \in \operatorname{Aut}(\mathcal{U})$ sending $b_{0}$ to $b_{1}$. Let $b_{n}=\sigma^{n}\left(b_{0}\right)$ (in particular, this is consistent with the naming of $b_{1}$ ). Then $\left\langle b_{i}: i\langle\omega\rangle\right.$ and $\psi$ is a witness to the (strict) order property, a contradiction to the fact that $T$ is stable. Therefore, there exists $\delta(y) \in p(y)$ such that, for all $b_{0}, b_{1} \in \mathcal{U}_{y_{i}}$ with $=\delta\left(b_{0}\right) \wedge \delta\left(b_{1}\right)$, either $\psi\left(\mathcal{U} ; b_{0}\right)=\psi\left(\mathcal{U} ; b_{1}\right)$ or $\psi\left(\mathcal{U} ; b_{0}\right) \cap \psi\left(\mathcal{U} ; b_{1}\right)=\emptyset$. In other words, the formula

$$
E_{\psi, p}\left(x_{0}, x_{1}\right):=(\exists y)\left(\delta(y) \wedge \psi\left(x_{0} ; y\right) \wedge \psi\left(x_{1} ; y\right)\right) \vee\left(x_{0}=x_{1}\right)
$$

is a $\emptyset$-definable equivalence relation. Now take

$$
\Psi:=\left\{E_{\psi, p} \mid \psi(x ; y) \in \Psi^{\prime}, p \in S_{y}(\emptyset)\right\} .
$$

We claim that $\Psi$ is a generating family for $T$. To show this, we simply show $\mathcal{C}_{\Psi}=\mathcal{C}_{\Psi^{\prime}}$. For $A \in \mathcal{C}_{\Psi^{\prime}}, A=\psi(\mathcal{U} ; b)$ for some $\psi(x ; y) \in \Psi^{\prime}, b \in \mathcal{U}_{y}$. Then, for any $a \in A$, one can check that $A=E_{\psi, \operatorname{tp}(b)}(\mathcal{U} ; a)$. Conversely, take $A \in \mathcal{C}_{\Psi}$, so $A=E_{\psi, p}(\mathcal{U}, a)$ for some $\psi(x ; y) \in \Psi^{\prime}, p \in S(\emptyset)$, and $a \in \mathcal{U}$. Let $\delta(y) \in p(y)$ be the associated formula. If there exists $b \in \mathcal{U}_{y}$ such that $\models \delta(b) \wedge \psi(a ; b)$, then $\psi(\mathcal{U} ; b)=A$, hence $A \in \mathcal{C}_{\Psi^{\prime}}$. On the other hand, if there exists no such $b$, then $A=\{a\}$ so, since $(x=y) \in \Psi^{\prime}, A \in \mathcal{C}_{\Psi^{\prime}}$.

So, without loss of generality, when dealing with a VC-minimal stable theory, we may assume the generating family is a set of equivalence relations on the home sort. As a corollary of Theorem 4.1, we get the following characterization of stable VC-minimal theories.

Theorem 5.2. Suppose $T$ is a stable theory in a countable language. The following are equivalent:

(1) $T$ is VC-minimal,

(2) for each formula $\varphi(x ; y)$, there exist finitely many refining definable equivalence relations $\left\{E_{j}\left(x_{0}, x_{1}\right) \mid j<m\right\}$, each of which is immortal, such that, for all $b \in \mathcal{U}_{y}, \varphi(\mathcal{U} ; b)$ is a (finite) boolean combination of instances of the $E_{j}$ 's. 

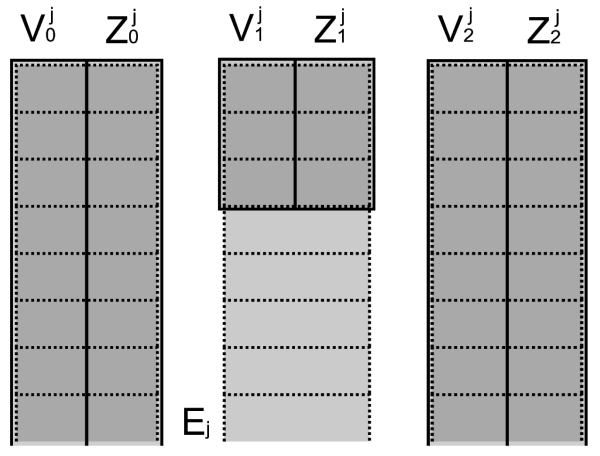

Figure 1. Example of construction where 1 enters $W_{g(i, j)}$ at stage 3.

\section{6. $\Pi_{4}^{0}$-COMPleteness OF VC-Minimality}

We now show that the characterization of VC-minimality given in Theorem 4.1 is the simplest possible.

Theorem 6.1. The index set of VC-minimal theories is $\Pi_{4}^{0}$-hard.

Proof. We describe a recursive function $f$, which, on a given input $i$, outputs a theory $T_{i}$ so that $T_{i}$ is always $\aleph_{0}$-stable, and $T_{i}$ is VC-minimal if and only if $i \in S$ for a $\Pi_{4}^{0}$-complete set $S$. We have $S$ written as $\forall j\left(W_{g(i, j)}\right.$ is co-finite $)$ for a fixed recursive function $g$.

Our theory will be in the language $L:=\left\{E_{j} \mid j \in \omega\right\} \cup\left\{U_{j} \mid j \in \omega\right\} \cup\left\{V_{k}^{j}, Z_{k}^{j} \mid\right.$ $j, k \in \omega\}$ where each $E_{j}$ is binary and all other relations are unary.

$T_{i}$ begins with the following axioms:

- The $U_{j}$ 's define disjoint infinite sets.

- Each $E_{j}$ is an equivalence relation on $U_{j}$ with infinitely many infinite classes.

- The $V_{k}^{j}$ 's define disjoint subsets of $U_{j}$.

- If $x \in V_{k}^{j}, y \in V_{l}^{j}$ for $k \neq l$, then $\neg E_{j}(x, y)$.

- For each $j, k \in \omega$ : There are infinitely many $E_{j}$-classes which do not intersect $V_{k}^{j}$.

- For each $j, k \in \omega$ : For each $E_{j}$-class $A$ which intersects $V_{k}^{j}$, both $A \cap V_{k}^{j}$ and $A \backslash V_{k}^{j}$ are infinite.

- For each $j, k \in \omega: x \in Z_{k}^{j}$ if and only if $x \notin V_{k}^{j}$ and there is a $y$ so that $E_{j}(x, y) \wedge y \in V_{k}^{j}$.

At stage $s$, for each $k \leq s$, we add the following axioms to $T_{i}$ :

- If $k \notin W_{g(i, j)}^{s}$, then add an axiom stating that there are at least $s E_{j}$-classes which intersect $V_{k}^{j}$.

- If $k$ enters $W_{g(i, j)}$ at stage $s$, add an axiom stating that there are exactly $s$ $E_{j}$-classes which intersect $V_{k}^{j}$.

See Figure 1 for details.

Lemma 6.2. For every $i, T_{i}$ is a complete $\aleph_{0}$-stable theory with quantifier elimination. 
Proof. For quantifier elimination, it suffices to show that we can eliminate $(\exists x)$ from a conjunction of formulae of the form $U_{j}(x), \pm E_{j}\left(x, y_{\ell}\right), \pm V_{k}^{j}(x)$, and $\pm Z_{k}^{j}(x)$ for some fixed $j$. As any $E_{j}$-class which intersects $V_{k}^{j}$ is contained in $V_{k}^{j} \cup Z_{k}^{j}$, this is straightforward. Moreover, a simple type-counting argument shows that $T_{i}$ is $\aleph_{0}$-stable.

Lemma 6.3. If $\forall j\left(W_{g(i, j)}\right.$ is co-finite $)$, then $T_{i}$ is VC-minimal.

Proof. For each $j$, let $S_{j}$ be the set $\omega \backslash W_{g(i, j)}$. Each $S_{j}$ is finite, by assumption. Define $X_{j}$ to be the set of elements in $U_{j}$, but not in any $V_{k}^{j}$ or $Z_{k}^{j}$ for $k \in S_{j}$. Let $\Phi$ be the family composed of the following families of definable sets:

- $\left\{U_{j} \mid j \in \omega\right\}$,

- $\left\{V_{k}^{j}, Z_{k}^{j} \mid k \in S_{j}, j \in \omega\right\}$,

- $\left\{X_{j} \mid j \in \omega\right\}$,

- $\left\{E_{j}(x, y) \wedge x \in V_{k}^{j} \mid k \in S_{j}\right\}$,

- $\left\{E_{j}(x, y) \wedge x \in Z_{k}^{j} \mid k \in S_{j}\right\}$,

- $\left\{E_{j}(x, y) \wedge x \in X_{j}\right\}$,

- $\left\{E_{j}(x, y) \wedge V_{l}^{j}(x) \mid l \notin S_{j}\right\}$,

- $\left\{E_{j}(x, y) \wedge Z_{l}^{j}(x) \mid l \notin S_{j}\right\}$.

It is immediate that $\Phi$ is directed. For $l \notin S_{j}, V_{l}^{j}$ is a finite union of instances of $\left\{E_{j}(x, y) \wedge V_{l}^{j}(x)\right\}$. A similar condition holds for $Z_{l}^{j}$. Each $E_{j}$-class is the union of elements of $\Phi$ given by the fourth, fifth, and sixth lines. By quantifier elimination, every definable set is a boolean combination of instances from $\Phi$. Thus $\Phi$ witnesses VC-minimality of $T_{i}$.

Lemma 6.4. If $\exists j\left(W_{g(i, j)}\right.$ is co-infinite $)$, then $T_{i}$ is non-VC-minimal.

Proof. Fix $j$ so $W_{g(i, j)}$ is co-infinite. Let $\psi$ be a directed formula so that every instance of $E_{j}$ is a boolean combination of instances of $\psi$. By Lemma 5.1, $\psi$ can be assumed to be comprised of equivalence relations. By quantifier elimination, instances of $\psi$ are $E_{j}$-classes away from finitely many exceptional $V_{k}^{j}$ and $Z_{k}^{j}$. Let $k \notin W_{g(i, j)}$ not be one of those finitely many exceptional $k$. Thus $V_{k}^{j}$ intersects infinitely many $E_{j}$-classes. This shows that $V_{k}^{j}$ devastates $\psi$. Thus $\psi$ cannot be contained in any family witnessing VC-minimality of $T_{i}$ by Lemma 3.7, and thus $T_{i}$ is non-VC-minimal.

Corollary 6.5. The index set of $V C$-minimal theories is $\Pi_{4}^{0}$-complete.

Remark 6.6. One should note that all the theories $T_{i}$ constructed in Theorem 6.1 are, in fact, convexly orderable. This gives us a large list of examples of theories that are $\aleph_{0}$-stable and convexly orderable but not VC-minimal.

\section{Complexity of DP-SMallness}

Definition 7.1. We say a theory $T$ is $d p$-small if there does not exist an $L$-formula $\varphi(x ; y)$, a sequence $\left\langle b_{i}: i<\omega\right\rangle$, and $L(\mathcal{U})$-formulae $\psi_{j}(x)$ (where $|x|=1$ ) such that, for all $i, j<\omega$, the following partial type is consistent with $T$ :

$$
\left\{\varphi\left(x ; b_{i}\right), \psi_{j}(x)\right\} \cup\left\{\neg \varphi\left(x ; b_{i^{\prime}}\right) \mid i^{\prime} \neq i\right\} \cup\left\{\neg \psi_{j^{\prime}}(x) \mid j^{\prime} \neq j\right\} .
$$




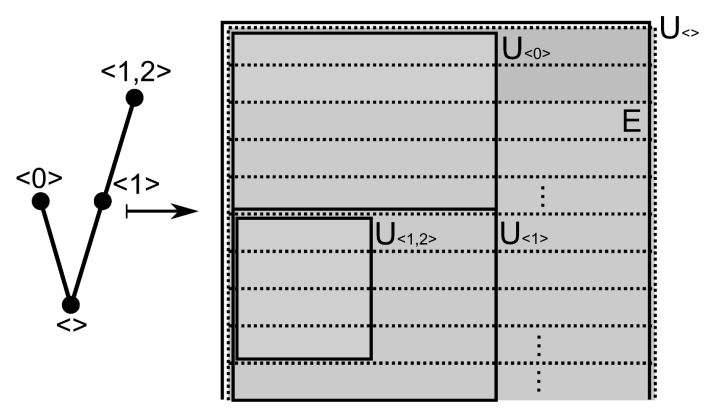

FiguRE 2. Example of construction of a particular tree.

By Proposition 1.5 of [6], if a theory is convexly orderable, then it is dp-small. In particular, all VC-minimal theories are dp-small.

A theory being not dp-small is clearly a $\Sigma_{1}^{1}$ condition, hence the index of dp-small theories is $\Pi_{1}^{1}$.

Theorem 7.2. The index set of $d p$-small theories is $\Pi_{1}^{1}$-complete.

Proof. We use the fact that $\left\{\mathcal{T} \subset \omega^{<\omega} \mid \mathcal{T}\right.$ is a recursive tree with no path $\}$ is $\Pi_{1}^{1}$ complete (see Theorem 5.14 of [3]). Given a (recursive index for a) tree $\mathcal{T} \subseteq \omega^{<\omega}$, we produce a theory so that the tree $\mathcal{T}$ has a path if and only if the theory is not dp-small. We fix the language $\mathcal{L}:=\{E\} \cup\left\{U_{\sigma} \mid \sigma \in \omega^{<\omega}\right\}$ where $E$ is binary and each $U_{\sigma}$ is unary.

The theory is axiomatized as follows:

- $E$ is an equivalence relation with infinitely many infinite classes.

- $\forall x U_{\emptyset}(x)$.

- If $\sigma$ and $\tau$ are incomparable, then $x \in U_{\sigma}$ and $y \in U_{\tau}$ implies $\neg E(x, y)$.

- If $\sigma \prec \tau$, then $U_{\tau} \subseteq U_{\sigma}$.

- If $\tau \notin \mathcal{T}$, then $U_{\tau}=\emptyset$.

- If $\tau=\sigma \frown\langle i\rangle$, and $\tau \in \mathcal{T}$, then there is an infinite set $S$ of $E$-equivalence classes so that for each $E$-equivalence class $A \in S, U_{\tau} \cap A$ is an infinite coinfinite subset of $U_{\sigma} \cap A$. Further, there are infinitely many $E$-equivalence classes which intersect $U_{\sigma}$ which do not intersect $U_{\tau}$.

See Figure 2 for an example.

It is straightforward to verify that the theory produced is complete for any $\mathcal{T}$ and is dp-small if and only if $\mathcal{T}$ has no infinite path.

For example, if there is an infinite path, say $\emptyset \prec \sigma_{0} \prec \sigma_{1} \prec \ldots$, take $\varphi(x ; y):=$ $E(x, y), \psi_{j}(x):=U_{\sigma_{j}}(x) \wedge \neg U_{\sigma_{j+1}}(x)$ for $j \in \omega$, and pairwise non- $E$-related $b_{i}$ 's outside of $U_{\sigma_{0}}$, each $E$-related to elements in $U_{\sigma_{j}}$ for all $j$. This is a witness to the non-dp-smallness of the theory.

To conclude this section, we use the ideas behind the construction in Theorem 6.1 to provide an answer to a question from [6].

Example 7.3. We give an example of a theory in a countable language that is dp-small but not convexly orderable, answering a question from [6]. This theory happens to be $\aleph_{0}$-stable. Let $L=\{E\} \cup\left\{U_{i, j} \mid j \leq i<\omega\right\}$, where $E$ is a binary 
relation and each $U_{i, j}$ is a unary relation. Let $T$ be the $L$-theory which says

- $E$ is an equivalence relation with infinitely many infinite classes;

- the $U_{i, j}$ are pairwise disjoint;

- for all $i<\omega, U_{i, 0} \cup \cdots \cup U_{i, i}$ is a union of infinitely many E-classes;

- if an $E$-class intersects $U_{i, j}$, it does so with infinitely many points and it intersects each $U_{i, j^{\prime}}$ for $j^{\prime} \leq i$; and

- if an $E$-class intersects $U_{i, j}$, it does not intersect $U_{i^{\prime}, j}$ for $i^{\prime} \neq i$.

This is $\aleph_{0}$-stable and has quantifier elimination.

Suppose, by means of contradiction, that it were convexly orderable, say with $\triangleleft$ on $M \models T$. Then, there exists $k<\omega$ such that, for all $a \in M, E(M ; a)$ is a union of at most $k \triangleleft$-convex sets. Look at $U_{2 k, j}$ for $j \leq 2 k$. Again, by convex orderability, there exists $\ell<\omega$ such that each $U_{2 k, j}(M)$ is a union of at most $\ell$ $\triangleleft$-convex sets. Let $B_{j, m}$ for $m<\ell$ be the $m$ th $\triangleleft$-convex component of $U_{2 k, j}(M)$ (some may be empty). By the pigeonhole principle, there exists $m_{0}, \ldots, m_{k}<\ell$ and an infinite collection of $E$-classes $A_{0}, A_{1}, \ldots$ such that $B_{j, m_{j}} \cap A_{t} \neq \emptyset$ for all $j \leq k$ and $t<\omega$. As the $B_{j, m_{j}}$ are $\triangleleft$-convex and pairwise disjoint, and each intersect $A_{0}$ and $M \backslash A_{0}$, we must have that $A_{0}$ is a union of at least $k+1 \triangleleft$-convex sets. This is a contradiction.

However, this theory is dp-small. Suppose, by means of contradiction, that $\varphi(x ; y)$ together with $\psi_{\ell}(x)$ for $\ell<\omega$ is a witness to non-dp-smallness. That is, there exists $\left\langle b_{i}: i<\omega\right\rangle$ such that, for all $i, \ell<\omega$, the partial type

$$
\left\{\varphi\left(x ; b_{i}\right), \psi_{\ell}(x)\right\} \cup\left\{\neg \varphi\left(x ; b_{i^{\prime}}\right) \mid i^{\prime} \neq i\right\} \cup\left\{\neg \psi_{\ell^{\prime}}(x) \mid \ell^{\prime} \neq \ell\right\}
$$

is consistent. By quantifier elimination, we may assume $\varphi$ is $E$ with perhaps a restriction to some $U_{i, j}$ and that the $\psi_{\ell}(x)$ are of the form $U_{i, j}$ perhaps restricted to an $E$-class. One checks that such formulae cannot make the above partial type consistent.

\section{The Main Construction Lemma}

Suppose $M$ is a countable model of a theory $T$ in a countable language. In this section, for simplicity of exposition, for a formula $\varphi(x ; y)$ and $b \in M_{y}$, we will write $\varphi_{b}$ to mean $\varphi(M ; b)$.

Lemma 8.1 (Unions and intersections of chains). Suppose $\rho(x ; y)$ and $\tau(x ; z)$ are so that for any $y, z, \rho_{y} \not \perp \tau_{z}$. Let $\chi$ be any union of a chain of instances of $\rho$ or intersection of a chain of instances of $\rho$. Then for every $z, \tau_{z} \not \perp \chi \chi$.

Proof. We first suppose $\chi$ is a union of a chain of instances of $\rho$. Suppose $z$ is so that $\tau_{z} \perp \chi$. Let $a$ be in the intersection and $b$ be in $\chi \backslash \tau_{z}$. Let $\rho_{w}$ be in the chain so that it contains $a$ and $b$. Then $\rho_{w} \perp \tau_{z}$, which is a contradiction.

Now suppose $\chi$ is an intersection of a chain of instances of $\rho$. Suppose $z$ is so that $\tau_{z} \perp \chi$. Let $a$ be any element of $\tau_{z} \backslash \chi$ and choose $w$ so $\rho_{w}$ does not contain $a$. Then $\rho_{w} \perp \tau_{z}$, which is a contradiction.

Lemma 8.2 (Main Construction Lemma). If $\varphi(x ; y)$ and $\psi(x ; z)$ are each a directed immortal formula (not assuming $\{\varphi, \psi\}$ is directed), then there exists $\delta(x ; w)$ an immortal formula such that

- $\{\psi, \delta\}$ is directed, and

- each instance of $\varphi$ is a finite boolean combination of instances of $\psi$ and $\delta$. 


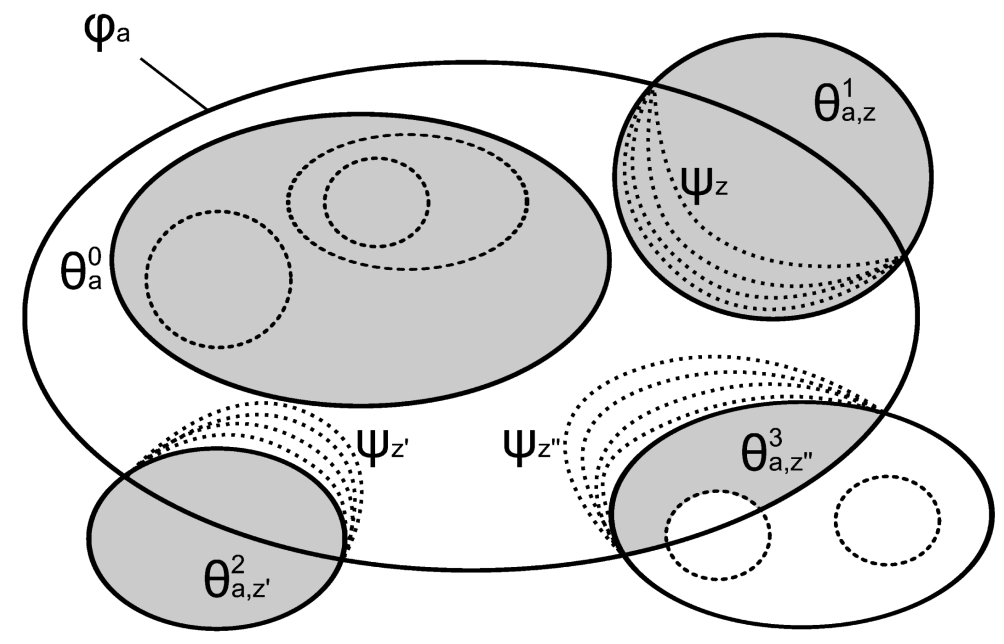

Figure 3. Example of $\theta^{0}, \theta^{1}, \theta^{2}$, and $\theta^{3}$.

Proof. We begin by defining the following formulae:

- $\theta_{a}^{0}:=\left\{x \in \varphi_{a} \mid \forall z\left(x \in \psi_{z} \rightarrow \psi_{z} \not \perp \varphi_{a}\right)\right\}$.

- If $\varphi_{a} \perp \psi_{z}$, then define $\theta_{a, z}^{1}:=\bigcup\left\{\psi_{y} \mid \psi_{y} \backslash \varphi_{a}=\psi_{z} \backslash \varphi_{a}\right\}$.

Otherwise, $\theta_{a, z}^{1}:=\emptyset$.

- If $\varphi_{a} \perp \psi_{z}$, then define $\theta_{a, z}^{2}:=\bigcap\left\{\psi_{y} \mid \psi_{y} \backslash \varphi_{a}=\psi_{z} \backslash \varphi_{a}\right\}$.

Otherwise, $\theta_{a, z}^{2}:=\emptyset$.

- $\theta_{a, z}^{3}:=\left(\theta_{a, z}^{2} \cap \varphi_{a}\right) \backslash \bigcup\left\{\psi_{z^{\prime}} \mid\left(\psi_{z^{\prime}} \perp \varphi_{a}\right) \wedge \psi_{z^{\prime}} \cap \varphi_{a} \subsetneq \theta_{a, z}^{2} \cap \varphi_{a}\right\}$.

See Figure 3 for an example.

We intend to show that $\left\{\psi(x ; y), \theta^{0}(x ; a), \theta^{1}(x ; a, z), \theta^{2}(x ; a, z), \theta^{3}(x ; a, z)\right\}$ is directed, each $\theta^{i}$ is immortal, and that each instance of $\varphi$ is a boolean combination of instances from this family.

Lemma 8.3. $\left\{\psi(x ; y), \theta^{0}(x ; a), \theta^{1}(x ; a, z), \theta^{2}(x ; a, z), \theta^{3}(x ; a, z)\right\}$ is directed.

Proof. For each pair of formulae from $\left\{\psi(x ; y), \theta^{0}(x ; a), \theta^{1}(x ; a, z), \theta^{2}(x ; a, z)\right.$, $\left.\theta^{3}(x ; a, z)\right\}$, we argue that no two instances can be $\perp$.

$\psi, \theta^{0}$ : If $x \in \psi_{y} \cap \theta_{a}^{0}$, then $\psi_{y} \not \perp \varphi_{a}$. If $\psi_{y} \supset \varphi_{a}$, then $\theta_{a}^{0} \subseteq \varphi_{a} \subseteq \psi_{y}$. So we suppose $\psi_{y} \subseteq \varphi_{a}$. Take any $x^{\prime} \in \psi_{y}$. If $x^{\prime}$ were in some $\psi_{y^{\prime}}$ where $\psi_{y^{\prime}} \perp \varphi_{a}$, then $x \in \psi_{y} \subseteq \psi_{y^{\prime}}$, contradicting $x \in \theta_{a}^{0}$. Thus $\psi_{y} \subseteq \theta_{a}^{0}$.

$\psi, \theta^{1}$ : This follows from Lemma 8.1 since any instance of $\theta^{1}$ is a union of a chain of instances of $\psi$ and $\psi$ is directed.

$\psi, \theta^{2}$ : This follows from Lemma 8.1 since any instance of $\theta^{2}$ is an intersection of a chain of instances of $\psi$ and $\psi$ is directed.

$\psi, \theta^{3}$ : Let $\psi_{y}$ intersect $\theta_{a, z}^{3}$. If $\psi_{y} \supseteq \psi_{z} \backslash \varphi_{a}$, then $\psi_{y} \supseteq \theta_{a, z}^{2} \supseteq \theta_{a, z}^{3}$. So we may assume $\psi_{y} \backslash \varphi_{a} \subsetneq \psi_{z} \backslash \varphi_{a}$. If for some $w, \psi_{w} \perp \varphi_{a}$ and $\psi_{w} \supseteq \psi_{y}$ and $\psi_{w} \subset \theta_{a, z}^{2}$, then $\psi_{y}$ is explicitly excluded from $\theta_{a, z}^{3}$ and the intersection is empty. Otherwise, $\psi_{y} \subseteq \theta_{a, z}^{2} \cap \varphi_{a}$ and it is contained in $\theta_{a, z}^{3}$. 
$\theta^{0}, \theta^{0}$ : We may assume $\varphi_{a^{\prime}} \subseteq \varphi_{a}$. If there is no $x \in \theta_{a^{\prime}}^{0}$ and $y$ so $x \in \psi_{y}$ and $\psi_{y} \perp \varphi_{a}$, then $\theta_{a^{\prime}}^{0} \subseteq \theta_{a}^{0}$. Otherwise, this $\psi_{y}$ must contain $\varphi_{a^{\prime}}$, since $\psi_{y} \not \perp \varphi_{a^{\prime}}$. Thus $\theta_{a^{\prime}}^{0} \subseteq \varphi_{a^{\prime}}$ and $\varphi_{a^{\prime}} \cap \theta_{a}^{0}=\emptyset$.

$\theta^{0}, \theta^{3}$ : Let $x \in \theta_{a}^{0} \cap \theta_{a^{\prime}, v}^{3}$. Let $S$ be the set of $v^{\prime}$ so that $\psi_{v} \backslash \varphi_{a^{\prime}}=\psi_{v^{\prime}} \backslash \varphi_{a^{\prime}}$. Then $x \in \psi_{v^{\prime}}$ for every $v^{\prime} \in S$. So $\psi_{v^{\prime}} \not \perp \varphi_{a}$ for each $v^{\prime} \in S$. If $\psi_{v^{\prime}} \subseteq \varphi_{a}$ for any $v^{\prime} \in S$, then $\psi_{v^{\prime}} \subseteq \theta_{a}^{0}\left(\right.$ see $\left.\psi, \theta^{0}\right)$, so $\theta_{a^{\prime}, v}^{3} \subseteq \psi_{v^{\prime}} \subseteq$ $\theta_{a}^{0}$. So we assume $\varphi_{a} \subseteq \psi_{v^{\prime}}$ for each such $v^{\prime}$. Thus $\varphi_{a} \subseteq \theta_{a^{\prime}, v}^{2}$. Thus $\theta_{a}^{0} \subseteq \varphi_{a} \subseteq \varphi_{a^{\prime}} \cap \theta_{a^{\prime}, v}^{2}$. If there is a $z$ so that $\theta_{a}^{0} \subseteq \psi_{z}$ and $\psi_{z} \perp \varphi_{a^{\prime}}$ and $\psi_{z} \cap \varphi_{a^{\prime}} \subsetneq \theta_{a^{\prime}, v}^{2} \cap \varphi_{a^{\prime}}$, then $\theta_{a}^{0} \cap \theta_{a^{\prime}, v}^{3}=\emptyset$. Otherwise, using the case $\psi, \theta_{0}$ above: $\theta_{a}^{0} \subseteq \theta_{a^{\prime}, v}^{3}$.

$\theta^{1}$, anything: Since no instance of $\psi$ is $\perp$ to any instance of a $\theta^{i}$, this follows by Lemma 8.1 .

$\theta^{2}$, anything: Since no instance of $\psi$ is $\perp$ to any instance of a $\theta^{i}$, this follows by Lemma 8.1 .

$\theta_{a, z}^{3}, \theta_{a^{\prime}, z^{\prime}}^{3}:$ As $\theta_{a, z}^{3} \subseteq \varphi_{a}$, we may assume $\varphi_{a} \supseteq \varphi_{a^{\prime}}$. Similarly, we may assume either $\theta_{a, z}^{2} \subseteq \theta_{a^{\prime}, z^{\prime}}^{2}$ or vice versa. We start with the first case: $\theta_{a, z}^{2} \subseteq$ $\theta_{a^{\prime}, z^{\prime}}^{2}$. Let $S$ be the set of $w$ so that $\psi_{w} \backslash \varphi_{a}=\psi_{z} \backslash \varphi_{a}$. Then for every $w \in S$, since $\psi_{w} \perp \varphi_{a}$, it follows that $\psi_{w} \perp \varphi_{a^{\prime}}$. Since $\left\{\psi, \theta^{2}\right\}$ is directed, either $\psi_{w}$ is a proper subset of $\theta_{a^{\prime}, z^{\prime}}^{2}$ and is thus excluded from $\theta_{a^{\prime}, z^{\prime}}^{3}$ or $\psi_{w}$ contains $\theta_{a^{\prime}, z^{\prime}}^{2}$. In the first case, $\theta_{a, z}^{3}$ is disjoint from $\theta_{a^{\prime}, z^{\prime}}^{3}$, so we suppose the second case holds for every $w \in S$. Thus $\theta_{a, z}^{2} \supseteq \theta_{a^{\prime}, z^{\prime}}^{2}$. It remains to check that any $\psi_{y}$ contained in $\theta_{a, z}^{2}$ excluded from $\theta_{a, z}^{3}$ is also excluded from $\theta_{a^{\prime}, z^{\prime}}^{3}$. If $\psi_{y} \perp \varphi_{a}$, then $\psi_{y} \perp \varphi_{a^{\prime}}$ and if it defines a proper subset of $\theta_{a^{\prime}, z^{\prime}}^{2} \cap \varphi_{a^{\prime}}$, then it defines a proper subset of $\theta_{a, z}^{2} \cap \varphi_{a}$, as needed.

Now we consider the second case: $\theta_{a^{\prime}, z^{\prime}}^{2} \subsetneq \theta_{a, z}^{2}$. If $\theta_{a^{\prime}, z^{\prime}}^{2} \cap \varphi_{a} \subsetneq$ $\theta_{a, z}^{2} \cap \varphi_{a}$, then using a small enough instance of $\psi_{w^{\prime}}$ where $\psi_{w^{\prime}} \backslash \varphi_{a^{\prime}}=$ $\psi_{z^{\prime}} \backslash \varphi_{a^{\prime}}$, we see that $\theta_{a^{\prime}, z^{\prime}}^{2}$ is excluded from $\theta_{a, z}^{3}$. Thus we may assume $\theta_{a^{\prime}, z^{\prime}}^{2} \cap \varphi_{a}=\theta_{a, z}^{2} \cap \varphi_{a}$. It remains to see that any instance of $\psi_{y}$ omitted from $\theta_{a, z}^{3}$ is also omitted from $\theta_{a^{\prime}, z^{\prime}}^{3}$. Since $\varphi_{a^{\prime}} \subseteq \varphi_{a}$, if $\psi_{y}$ intersects $\theta_{a^{\prime}, z^{\prime}}^{3}$ and $\psi_{y} \perp \varphi_{a}$, then $\psi_{y} \perp \varphi_{a^{\prime}}$. Thus if $\psi_{y}$ is omitted in the definition of $\theta_{a, z}^{3}$, it is also omitted in the definition of $\theta_{a^{\prime}, z^{\prime}}^{3}$. Thus $\theta_{a^{\prime}, z^{\prime}}^{3} \subseteq \theta_{a, z}^{3}$.

Lemma 8.4. Suppose $\rho(x ; y)$ is an immortal formula, and that each instance of $\chi(x ; z)$ is a union of a chain of instances of $\rho$. Then $\chi$ is immortal.

Suppose $\rho(x ; y)$ is an immortal formula, and that each instance of $\chi(x ; z)$ is an intersection of a chain of instances of $\rho$. Then $\chi$ is immortal.

Proof. First we consider the case where every instance of $\chi(x ; z)$ is a union of a chain of instances of $\rho$. Suppose towards a contradiction that $\gamma(x)$ devastates $\chi$ witnessed by the indiscernible $\left\langle c_{i}: i<\omega\right\rangle$. For all $i<j<\omega, \chi_{c_{i}} \backslash \chi_{c_{j}}$ intersects both $\gamma$ and $\neg \gamma$. Since each $\chi$ instance is a union of a chain of instances of $\rho$, there exists $d_{i}$ for each $i<\omega$ so that $\rho_{d_{i}} \subseteq \chi_{c_{i}}$ and for all $i<j, \rho_{d_{i}} \backslash \chi_{c_{j}}$ intersects both $\gamma$ and $\neg \gamma$. This witnesses that $\gamma$ devastates $\rho$, contrary to the assumption of $\rho$ 's immortality. 
Now we consider the case where every instance of $\chi(x ; z)$ is an intersection of a chain of instances of $\rho$. Suppose towards a contradiction that $\gamma(x)$ devastates $\chi$ witnessed by the indiscernible $\left\langle c_{i}: i<\omega\right\rangle$. For all $i<j<\omega, \chi_{c_{i}} \backslash \chi_{c_{j}}$ intersects both $\gamma$ and $\neg \gamma$. Since each $\chi$ instance is an intersection of a chain of instances of $\rho$, there exists $d_{i}$ for each $i<\omega$ so that $\chi_{c_{i}} \subseteq \rho_{d_{i}}$ and for all $i<j, \rho_{d_{i}} \backslash \rho_{d_{j}}$ intersects both $\gamma$ and $\neg \gamma$. This witnesses again that $\gamma$ devastates $\rho$, contrary to the assumption of $\rho$ 's immortality.

Lemma 8.5. $\theta^{0}$ is immortal.

Proof. Towards a contradiction, suppose $\gamma$ devastates $\theta^{0}$ and consider the indiscernible sequence $\left\langle a_{i}: i<\omega\right\rangle$ witnessing this as in Lemma 3.3. If $\varphi_{a_{0}} \cap \varphi_{a_{1}}=\emptyset$, then $\gamma$ devastates $\varphi$ by indiscernibility, contradicting the immortality of $\varphi$. If $\varphi_{a_{0}} \subseteq \varphi_{a_{1}}$, then one of two cases holds:

(i) There exists $z$ such that $\theta_{a_{0}}^{0} \subseteq \psi_{z}$ and $\psi_{z} \perp \varphi_{a_{1}}$. In this case, $\theta_{a_{0}}^{0}$ and $\theta_{a_{1}}^{0}$ are disjoint and $\theta_{a_{1}}^{0} \subseteq\left(\varphi_{a_{1}} \backslash \varphi_{a_{0}}\right)$. Therefore, by indiscernibility, $\gamma$ devastates $\varphi$, contrary to assumption.

(ii) There exists no such $z$. Then $\theta_{a_{0}}^{0} \subseteq \theta_{a_{1}}^{0}$, but this contradicts the choice of the $a_{i}$ 's in Lemma 3.3 .

Similarly, if $\varphi_{a_{1}} \subseteq \varphi_{a_{0}}$ and there exists $z$ such that $\theta_{a_{1}}^{0} \subseteq \psi_{z}$ and $\psi_{z} \perp \varphi_{a_{0}}$, then this contradicts the immortality of $\varphi$. Therefore, we must have that $\varphi_{a_{1}} \subseteq \varphi_{a_{0}}$ and no such $z$ exists. Hence $\theta_{a_{1}}^{0} \subseteq \theta_{a_{0}}^{0}$.

As $\gamma$ does not devastate $\varphi$, we must have that $\left(\varphi_{a_{0}} \backslash \varphi_{a_{1}}\right)$ is contained in either $\gamma$ or $\neg \gamma$. Without loss of generality, suppose it is contained in $\gamma$. Then, by indiscernibility, $\left(\varphi_{a_{i}} \backslash \varphi_{a_{i+1}}\right) \subseteq \gamma$ for all $i<\omega$.

Notice that $\neg \gamma \cap\left(\theta_{a_{0}}^{0} \backslash \theta_{a_{1}}^{0}\right) \neq \emptyset$ by assumption, so choose $x$ in this set. As $x \notin \theta_{a_{1}}^{0}$, there exists $z$ such that $x \in \psi_{z}$ and $\psi_{z} \perp \varphi_{a_{1}}$, hence $\psi_{z} \cap \theta_{a_{1}}^{0}=\emptyset$. However, since $x \in \theta_{a_{0}}^{0}$ and $\left\{\psi, \theta^{0}\right\}$ is directed, we must have that $\psi_{z} \subseteq \theta_{a_{0}}^{0}$. Therefore, $\psi_{z} \subseteq\left(\theta_{a_{0}}^{0} \backslash \theta_{a_{1}}^{0}\right)$. Moreover, as $\psi_{z} \perp \varphi_{a_{1}}$ and $\psi_{z} \subseteq \varphi_{a_{0}}$, we have that $\psi_{z} \cap\left(\varphi_{a_{0}} \backslash \varphi_{a_{1}}\right) \neq \emptyset$. Hence, $\psi_{z} \cap \gamma \neq \emptyset$. By indiscernibility, there are $z_{i}$ such that

- $\psi_{z_{i}} \cap \neg \gamma \neq \emptyset$,

- $\psi_{z_{i}} \cap \gamma \neq \emptyset$, and

- $\psi_{z_{i}} \subseteq\left(\theta_{a_{i}}^{0} \backslash \theta_{a_{i+1}}^{0}\right)$.

In particular, the $\psi_{z_{i}}$ 's are disjoint. Hence $\gamma$ devastates $\psi$, contrary to immortality of $\psi$.

Lemma 8.6. $\theta^{1}$ is immortal

Proof. This follows from Lemma 8.4

Lemma 8.7. $\theta^{2}$ is immortal

Proof. This follows from Lemma 8.4

Lemma 8.8. $\theta^{3}$ is immortal.

Proof. For this proof, let

$$
\theta_{a, b, z}^{4}:=\left(\theta_{a, z}^{2} \cap \varphi_{b}\right) \backslash \bigcup\left\{\psi_{z^{\prime}} \mid\left(\psi_{z^{\prime}} \perp \varphi_{b}\right) \wedge \psi_{z^{\prime}} \cap \varphi_{b} \subsetneq \theta_{a, z}^{2} \cap \varphi_{b}\right\} .
$$

In particular, $\theta_{a, a, z}^{4}=\theta_{a, z}^{3}$, so it suffices to show $\theta^{4}$ is immortal. 
By means of contradiction, suppose $\gamma$ devastates $\theta^{4}$, and consider the indiscernible sequence $\left\langle\left\langle a_{i}, b_{i}, z_{i}\right\rangle: i<\omega\right\rangle$ witnessing this as in Lemma 3.3. Fix any $i \neq j$. Since $\theta_{2}$ is directed, we have three cases:

(i) $\theta_{a_{i}, z_{i}}^{2} \cap \theta_{a_{j}, z_{j}}^{2}=\emptyset$,

(ii) $\theta_{a_{i}, z_{i}}^{2} \subseteq \theta_{a_{j}, z_{j}}^{2}$, or

(iii) $\theta_{a_{j}, z_{j}}^{2} \subseteq \theta_{a_{i}, z_{i}}^{2}$.

For Case (i), since $\gamma$ devastates $\theta^{4}$ and $\theta_{a_{i}, b_{i}, z_{i}}^{4} \subseteq \theta_{a_{i}, z_{i}}^{2}$, we have that $\gamma$ devastates $\theta^{2}$ by indiscernibility. Case (ii) and (iii) are symmetric, so let us suppose that Case (ii) holds. In almost the exact same way as one shows that $\psi_{y} \not \perp \theta_{a, z}^{3}$ for any $a, y, z$, one can show that $\psi_{y} \not \perp \theta_{a, b, z}^{4}$ for any $a, b, y, z$. Hence $\theta_{c, y}^{2} \not \perp \theta_{a, b, z}^{4}$ for any $a, b, c, y, z$ by Lemma 8.1. Thus there are three subcases:

(a) $\theta_{a_{i}, z_{i}}^{2} \cap \theta_{a_{j}, b_{j}, z_{j}}^{4}=\emptyset$,

(b) $\theta_{a_{i}, z_{i}}^{2} \subseteq \theta_{a_{j}, b_{j}, z_{j}}^{4}$, or

(c) $\theta_{a_{j}, b_{j}, z_{j}}^{4} \subseteq \theta_{a_{i}, z_{i}}^{2}$.

In Case (a), $\left(\theta_{a_{j}, b_{j}, z_{j}}^{4} \backslash \theta_{a_{i}, b_{i}, z_{i}}^{4}\right) \subseteq\left(\theta_{a_{j}, z_{j}}^{2} \backslash \theta_{a_{i}, z_{i}}^{2}\right)$, therefore $\gamma$ devastates $\theta^{2}$ by indiscernibility. In Case (b), fix $k<i<j$ or $k>i>j$. Then, by indiscernibility, $\theta_{a_{k}, d_{k}}^{2} \subseteq \theta_{a_{i}, b_{i}, z_{i}}^{4}$ and, by definition, $\theta_{a_{j}, b_{j}, z_{j}}^{4} \subseteq \theta_{a_{j}, z_{j}}^{2}$. Hence

$$
\left(\theta_{a_{j}, b_{j}, z_{j}}^{4} \backslash \theta_{a_{i}, b_{i}, z_{i}}^{4}\right) \subseteq\left(\theta_{a_{j}, z_{j}}^{2} \backslash \theta_{a_{k}, d_{k}}^{2}\right) .
$$

Therefore, $\gamma$ devastates $\theta^{2}$ by indiscernibility. Hence Case (c) must hold. Together, (ii) and (c) imply $\theta_{a_{i}, b_{j}, z_{i}}^{4}=\theta_{a_{j}, b_{j}, z_{j}}^{4}$. Hence, by indiscernibility, we may assume there are $a$ and $z$ such that, for all $i<\omega, \theta_{a, b_{i}, z}^{4}=\theta_{a_{i}, b_{i}, z_{i}}^{4}$. We now consider the sequence $\left\langle\left\langle a, b_{i}, z\right\rangle: i<\omega\right\rangle$ which witnesses that $\theta^{4}$ is devastated by $\gamma$.

If $\varphi_{b_{0}} \cap \varphi_{b_{1}}=\emptyset$, then, as $\theta_{a, b_{i}, z}^{4} \subseteq \varphi_{b_{i}}$ for all $i, \gamma$ devastates $\varphi$. So we may assume that $\varphi_{b_{1}} \subseteq \varphi_{b_{0}}$ (note that if $\varphi_{b_{0}} \subseteq \varphi_{b_{1}}$, then $\theta_{a, b_{0}, z}^{4} \subseteq \theta_{a, b_{1}, z}^{4}$, contrary to this sequence witnessing devastation of $\theta^{4}$ ). If both $\gamma$ and $\neg \gamma$ intersect $\varphi_{b_{0}} \backslash \varphi_{b_{1}}$, then $\gamma$ devastates $\varphi$. So, without loss of generality (and by indiscernibility), we may assume $\varphi_{b_{i}} \backslash \varphi_{b_{i+1}} \subseteq \gamma$ for all $i<\omega$. In particular, note that $\neg \gamma$ must intersect $\bigcap_{i<\omega} \varphi_{b_{i}}$.

Since $\neg \gamma$ intersects $\theta_{a, b_{0}, z}^{4} \backslash \theta_{a, b_{1}, z}^{4}$, there exists $w$ such that

- $\psi_{w}$ intersects $\neg \gamma$,

- $\psi_{w} \subseteq \theta_{a, z}^{2}$,

- $\psi_{w} \perp \varphi_{b_{1}}$, and

- $\psi_{w} \subseteq \varphi_{b_{0}}$.

In particular, $\psi_{w}$ intersects $\left(\varphi_{b_{0}} \backslash \varphi_{b_{1}}\right)$, hence also $\gamma$. For all $1 \leq i<\omega, \psi_{w}$ does not contain $\left(\varphi_{b_{i}} \backslash \varphi_{b_{i+1}}\right)$ as otherwise $\theta_{a, b_{i}, z}^{4}=\theta_{a, b_{i+1}, z}^{4}$, contrary to the choice of $b_{i}$. On the other hand, for all but finitely many $i, \psi_{w}$ does not intersect $\left(\varphi_{b_{i}} \backslash \varphi_{b_{i+1}}\right)$, as otherwise $\psi_{w}$ would devastate $\varphi$. By removing finitely many and reindexing, we may assume $\psi_{w}$ is disjoint from $\left(\varphi_{b_{i}} \backslash \varphi_{b_{i+1}}\right)$ for all $i>1$.

By indiscernibility, for each $i<\omega$, there exists $w_{i}$ such that

- $\psi_{w_{i}}$ intersects $\gamma$ and $\neg \gamma$,

- $\psi_{w_{i}}$ intersects $\left(\varphi_{b_{2 i}} \backslash \varphi_{b_{2 i+1}}\right)$, and

- $\psi_{w_{i}}$ is disjoint from $\left(\varphi_{b_{2 k}} \backslash \varphi_{b_{2 k+1}}\right)$ for all $k \neq i$.

(The last condition is clear for $k>i$ and, for $k<i$, note that $\psi_{w_{i}} \subseteq \varphi_{b_{2 i}}$, hence $\psi_{w_{i}}$ is disjoint from $\left(\varphi_{b_{2 k}} \backslash \varphi_{b_{2 k+1}}\right)$.) In particular, since $\psi$ is directed, the last 
two conditions imply that the $\psi_{w_{i}}$ 's are disjoint. Hence, by the first condition, $\gamma$ devastates $\psi$, contrary to immortality of $\psi$.

Lemma 8.9. For any $c, \varphi_{c}$ is a boolean combination of instances from $\left\{\psi, \theta^{0}, \theta^{1}\right.$, $\left.\theta^{2}, \theta^{3}\right\}$.

Proof. Every element in $\varphi_{c}$ is either in $\theta_{c}^{0}$ or is in some $\theta_{c, z}^{1}$. We first note that there is a finite set of instances of $\theta_{c, z}^{1}$ which suffices to cover $\left(\varphi_{c} \backslash \theta_{c}^{0}\right)$. Otherwise, we could choose more and more instances of $\theta_{c, z}^{1}$ which would witness that $\varphi_{c}$ devastates $\theta^{1}$.

We now define a sequence of sets whose union will be $\varphi_{c}$. Set $Y_{0}=\theta_{c}^{0}$. Suppose we have defined the sets $Y_{j}$ for $j<i$. Suppose further that there is a finite set $S_{i-1}$ of elements so that $\left(\bigcup_{w \in S_{i-1}} \theta_{c, w}^{1} \cap \varphi_{c}\right)=\left(\varphi_{c} \backslash \bigcup_{j<i} Y_{j}\right)$. Now we define

$$
Y_{i}:=\bigcup_{w \in S_{i-1}}\left(\left(\theta_{c, w}^{1} \backslash \theta_{c, w}^{2}\right) \cup \theta_{c, w}^{3}\right)
$$

To complete the recursive definition of the sequence of sets $Y_{i}$ for $i<\omega$, we need to see that there is a finite set $S_{i}$ so that $\left(\bigcup_{w \in S_{i}} \theta_{c, w}^{1} \cap \varphi_{c}\right)=\left(\varphi_{c} \backslash \bigcup_{j \leq i} Y_{j}\right)$. We build $S_{i}$ : Having selected elements $a_{0}, \ldots, a_{k-1}$ so that $\left(\bigcup_{j<k} \theta_{c, a_{j}}^{1} \cap \varphi_{c}\right) \subsetneq\left(\varphi_{c} \backslash \bigcup_{j \leq i} Y_{j}\right)$, we need to select an element $a_{k}$. Fix an element $x \in \varphi_{c} \backslash\left(\bigcup_{j \leq i} Y_{j} \cup \bigcup_{j<k} \theta_{c, a_{j}}^{1}\right)$ and let $a_{k}$ be an element so $x \in \theta_{c, a_{k}}^{1}$. By directedness of $\left\{\theta^{0}, \theta^{1}, \theta^{3}\right\}, \theta_{c, a_{k}}^{1} \cap \varphi_{c} \subseteq$ $\left(\bigcup_{j \leq i} Y_{j} \cup \bigcup_{j<k} \theta_{c, a_{j}}^{1}\right)$. This process must stop, yielding a finite set $S_{i}$, as otherwise $\varphi_{c}$ devastates $\theta^{1}$.

It remains to see that for some $i, \bigcup_{j \leq i} Y_{j}=\varphi_{c}$. Otherwise there is an infinite sequence of $b_{i}$ for $i \in \omega$ so that $b_{i} \in S_{i}$ for each $i$ and $\theta_{c, b_{i+1}}^{1} \subseteq \theta_{c, b_{i}}^{1}$, and this sequence witnesses that $\varphi$ devastates $\theta^{1}$.

As each $Y_{j}$ is a boolean combination of instances from $\left\{\psi, \theta^{0}, \theta^{1}, \theta^{2}, \theta^{3}\right\}, \varphi_{c}$ is a boolean combination of instances from $\left\{\psi, \theta^{0}, \theta^{1}, \theta^{2}, \theta^{3}\right\}$.

\section{REFERENCES}

[1] H. Adler, Theories controlled by formulas of Vapnik-Chervonenkis codimension 1 (2008). Preprint, http://www.logic.univie.ac.at/ adler/docs/vcm.pdf.

[2] U. Andrews, S. Cotter, J. Freitag, and A. Medvedev, VC-minimality: examples and observations (2014). Preprint.

[3] C. J. Ash and J. Knight, Computable structures and the hyperarithmetical hierarchy, Studies in Logic and the Foundations of Mathematics, vol. 144, North-Holland Publishing Co., Amsterdam, 2000. MR1767842(2001k:03090)

[4] J. Flenner and V. Guingona, Canonical forests in directed families, Proc. Amer. Math. Soc. 142 (2014), no. 6, 1849-1860, DOI 10.1090/S0002-9939-2014-11935-6. MR3182006

[5] J. Flenner and V. Guingona, Convexly orderable groups and valued fields, J. Symb. Log. 79 (2014), no. 1, 154-170, DOI 10.1017/jsl.2013.16. MR3226016

[6] V. Guingona, On VC-minimal fields and dp-smallness, Arch. Math. Logic 53 (2014), no. 5-6, 503-517, DOI 10.1007/s00153-014-0376-9. MR3237864 
[7] V. Guingona and M. C. Laskowski, On VC-minimal theories and variants, Arch. Math. Logic 52 (2013), no. 7-8, 743-758, DOI 10.1007/s00153-013-0341-z. MR3115992

Department of Mathematics, University of Wisconsin, Madison, Wisconsin 53706-1388

E-mail address: andrews@math.wisc.edu

URL: http://www.math.wisc.edu/ andrews/

Department of Mathematics, University of Notre Dame, 255 Hurley, Notre Dame, INDIANA 46556

Current address: Department of Mathematics, Ben-Gurion University of teh Negev, Be'er Sheva, Israel 8410501

E-mail address: guingona@math.bgu.ac.il

URL: http://www.math.bgu.ac.il/ guingona/ 